Zacklad, M. (2020). Les enjeux de la transition numérique et de l'innovation collaborative dans les mutations du travail et du management dans le secteur public. Dans A. Gillet (Éd.), Les transformations du travail dans les services publics. Presses de l'EHESP (preprint)

\title{
Les enjeux de la transition numérique et de l'innovation collaborative dans les mutations du travail et du management dans le secteur public
}

Manuel Zacklad (Professeur du Cnam en sciences de l'information et de la communication, Dicen-IdF)

\section{Résumé}

En partant d'une description des bouleversements introduits par la transition numérique et des technologies capacitantes qu'elle promeut, nous montrons comment les opérateurs sont parfois en demande de plus d'innovations numériques pour améliorer leurs conditions de travail et les services rendus au public, pour autant que celles-ci ne soient pas substitutives et excessivement rationnalisantes. Les démarches d'innovation collaborative soutenues, de manière en partie paradoxale, par le haut management contribuent à faciliter ces mutations.

\section{Introduction}

La transformation numérique et l'innovation collaborative ou les notions associées " d'intelligence collective », de « design thinking », " d'agilité » sont en passe de devenir les principaux concepts à la mode du management dans les organisations privées et publiques, au moins au sein des sièges et des directions centrales. En partant d'une description des spécificités des bouleversements introduits par la transition numérique et des technologies capacitantes qu'elle promeut, nous montrons comment les opérateurs sont parfois en demande de plus d'innovations numériques pour améliorer leurs conditions de travail et les services rendus au public, pour autant que celles-ci ne soient pas substitutives et excessivement rationnalisantes. Les démarches d'innovation collaborative soutenues de manière en partie paradoxale par le haut management contribuent à faciliter ces mutations.

\section{1-Transformation numérique}

Les transformations profondes des organisations et du travail de ces quarante dernières années ne peuvent être appréhendées sans une prise en compte du développement sans précédent des technologies de l'information et de la communication basée sur l'informatique ou plus généralement sur le numérique. D'un point de vue terminologique, quand nous parlerons du numérique ou des technologies numériques cela correspondra à ce que l'on avait coutume d'appeler les technologies de l'information et de la communication.

Quand nous parlons ici de transformation numérique ou de transition numérique (ou transformation digitale dans les entreprises privées pratiquant le " globish ») nous désignons un processus de transformation des métiers via des applications légères, souvent basées sur l'informatique en nuage (cloud) et en partie indépendantes du Système d'Information traditionnel et de ses progiciels de gestion intégrés (ERP), mais surtout d'une démarche qui donne le pouvoir aux métiers et aux utilisateurs dans des logiques dites «agiles» ou « collaboratives ».

L'agilité caractérise à l'origine les techniques de programmation et de déploiement en rendant possible des cycles de développement rapide permettant des interactions fréquentes entre les développeurs et des feed-back rapides des utilisateurs. Comme c'est souvent le cas, certains consultants ont ensuite élargi la notion pour parler de management ou d'organisation agile en 
faisant perdre à la notion une partie de sa substance. De manière plus fondée, l'agilité décrit souvent aujourd'hui un mode de conduite de projets, même quand ceux-ci ne relèvent pas directement de la programmation.

Le collaboratif est également un terme assez vague et englobant qui est notamment opposé dans certains textes à la notion de coopération par des consultants ou des auteurs assez mal informés. Dans le cadre de ce texte, nous le mobilisons pour appréhender les démarches « d'innovation collaborative » dont nous proposons une définition assez précise. Les démarches d'innovation collaborative ou de co-design regroupent un nombre assez vaste de méthodes et à ce titre, peuvent inclurent des éléments de méthodes agiles mais également de design thinking, de « creative problem solving », etc.

Si les inventions technologiques mais aussi leur maitrise, leur diffusion, leur appropriation et les détournements associés ont toujours été aux cœur des innovations des produits, des services et des procédés, les développements du numérique ont été à l'origine d'une multiplication sans précédent de ces innovations et ceci d'autant plus qu'il s'agit de technologies très accessibles aux innovateurs externes comme internes en comparaison d'autres technologies dans les domaines des matériaux, de l'énergie ou des sciences du vivant pour ne prendre que quelques exemples.

Intrinsèquement les technologies numériques sont en mesure de « lire » des signaux ou des symboles, de les stocker, puis de les "réécrire » selon des combinaisons différentes de celles qui prévalaient lors de la lecture. Entre la lecture et la réécriture, les signes font l'objet de traitements décrits par des algorithmes. Contrairement à ce que laissent entendre certains thuriféraires mal informés de l'Intelligence Artificielle (IA), les programmes n'ont accès à aucune signification, que ce soit dans les opérations de "lecture», de traitement ou "d'écriture ». Ils ne font que suivre des règles formelles qui reproduisent ou qui simulent des calculs et des inférences. Les programmes d'IA actuels font de la « reconnaissance de formes », sans aucune compréhension des contenus mais de manière d'autant plus sophistiquée que les ensembles de données sont importants (big data).

Cependant, l'utilisation des techniques d'IA dans les applications en lien avec des interfaces humain-machine de plus en plus puissantes (« reconnaissance» partielle des textes, de la parole, des gestes et du visage, génération approximative mais réaliste de textes et de paroles sur la base de données stockées, etc.) produisent des effets de sens majeurs pour les utilisateurs de ces technologies. La reconnaissance de formes est aussi susceptible de permettre un contrôle redoutable des activités individuelles et collectives quand les libertés des citoyens ne sont pas protégées par des règles strictes. Par ailleurs, le couplage des technologies numériques avec des capteurs électroniques et des actionneurs de machines permettent de réaliser des systèmes de production totalement automatisés pour le contrôle de processus ou la fabrication en série dans le monde industriel, mais aussi de manière plus récente, dans le monde du tertiaire.

\section{2-Technologies numériques substitutive, rationnalisante et capacitante}

Du point de vue de la nature du travail, la distinction la plus importante entre les technologies numériques est celle qui sépare les technologies inscrites dans une logique substitutive, rationnalisante ou capacitante (Zacklad, 2017), une typologie qui rejoint celle d'E. Bobilier Chaumon (2017). Quand l'informatisation se déroule de manière substitutive, les opérateurs qui réalisaient les tâches sont pour l'essentiel remplacés et peuvent, pour un petit nombre d'entre eux, se voir attribuer de nouveaux rôles de supervision ou de maintenance. Dans le 
domaine du tertiaire, quand cette logique est privilégiée, les progrès récents de l'IA auxquels nous avons fait référence, peuvent ainsi réduire le nombre d'emplois de contact avec le client au bénéfice d'emplois qui supervisent les interactions semi-automatisées. Ce remplacement n'est possible que dans le cadre d'une vision de la relation client assez éloignée de l'idéal de la co-production servicielle à valeur ajoutée, mais la tentation est présente dans les secteurs dont les offres sont très standardisées.

Dans les technologies numériques rationnalisantes, l'informatique est au service d'une poursuite de la standardisation taylorienne. L'humain ne peut être totalement remplacé par des automatismes notamment $\mathrm{du}$ fait de ses compétences gestuelles, communicationnelles et relationnelles... Mais si l'informatique ne remplace pas les opérateurs il impose des "mécanismes de coordination» souvent rigides et fortement prescriptifs. Ces technologies correspondent typiquement aux progiciels de gestion intégrés (ERP), aux workflows, aux logiciels utilisés dans les centres d'appel. Ces technologies peuvent avoir des effets délétères sur la santé des opérateurs du fait du caractère répétitif et contraignant des tâches qui leur sont reléguées.

Les technologies numériques capacitantes, au sens où P. Falzon (2013) utilise ce terme, sont caractéristiques de la transition numérique telle que nous l'envisageons, centrée sur des applications dont le développement est piloté par les métiers et dont l'utilisation ne peut pas être imposée de manière stricte. Si elles relèvent bien d'une forme "d'augmentation » des capacités, cette augmentation ne relève pas des fantasmes du transhumanisme ou de certaines applications à vocation médicale inscrites dans une logique palliative (Bobilier, Chaumon, 2017), qui correspondrait pour nous à une amplification symbiotique qui accroit de manière spécialisé la puissance d'un organe ou d'une fonction. Par opposition, l'augmentation capacitante ou apprenante développe le pouvoir d'agir et les compétences des opérateurs dans une perspective d'instrument psychologique (Vygotsky, 1992). Elle ne cherche pas à conditionner l'utilisateur ou à détourner son attention. Son appropriation est basée sur sa malléabilité qui permet son intégration dans «l'environnement support » (Zacklad, 2012).

Ces applications correspondent, aux outils d'aide à la conception dans le domaine de la publication, de l'architecture ou de l'ingénierie ou à des outils d'aide à la communication et à la coopération comme les Réseaux Sociaux d'Entreprise (RSE). Dans le domaine de la coopération, alors que les technologies rationnalisantes s'appuient sur des documents numériques étroitement codifiés, comme des formulaires de workflow, les technologies capacitantes s'appuient sur des « documents pour l'action» (messagerie, wiki, RSE, éditeurs de textes ou d'images...) qui coordonnent l'action tout en laissant une liberté d'ajustement (Zacklad, 2007).

Cette liberté « d'écriture » dans les documents pour l'action permet que se mettent en place des régulations ${ }^{1}$ dans le travail coopératif où la prise d'initiatives est possible tant dans le cadrage du déroulement de l'activité que dans la caractérisation des productions intermédiaires et finales. Ces initiatives s'inscrivent dans un travail de conception à part entière ou dans un travail de production créatif de nature servicielle ou artisanale. La régulation peut être managériale ou horizontale et correspond aux environnements où prédominent les prescriptions faibles et réciproques. La prescription faible «consiste à fixer non pas des buts, des missions ou des procédures, mais des «objets de travail » qui sont aussi des «objets de connaissance »: ils permettent une première orientation et une répartition provisoire des tâches. » (Hatchuel, 2002).

\footnotetext{
${ }^{1}$ Dans notre texte de 2007 nous distinguons transaction primaire et transaction régulatrice.
} 


\section{3-Relation particulière des opérateurs aux technologies numériques capacitantes}

Dans le domaine des technologies capacitantes le rapport des opérateurs à l'innovation technique est très différent de ce qu'il est dans les technologies substitutives ou rationnalisantes car "l'innovation technologique peut tout aussi bien être au service d'une amplification des capacités qui accroit l'inventivité, l'autonomie, la mémoire, la socialité des utilisateurs. » (Zacklad, 2012). Bien souvent, ce que revendiquent les utilisateurs des applications capacitantes " ce n'est pas moins d'innovation et moins de technologie, posture qui a pu être celle d'une résistance ouvrière à l'automatisation substitutive, mais d'avantage de technologies et d'innovations qui augmentent leur capacité d'agir et leur inventivité, leurs aptitudes à l'organisation et à la relation. ».

Le discours de ces collaborateurs est de considérer que le système d'information traditionnel, dont les applications relèvent de technologies rationnalisantes, est rétrograde par rapport aux applications qu'ils expérimentent dans d'autres contextes et notamment dans leur vie privée. Ils souhaitent utiliser leurs terminaux personnels au travail et considèrent que les applications disponibles les brident dans leur pouvoir d'agir à l'intérieur de l'organisation comme dans la relation avec les parties prenantes externes dont les clients ou les usagers.

Pour peu qu'ils soient un peu familiarisés avec les usages avancés du numérique, les innovateurs du quotidien (cf. supra) imaginent sans peine des innovations de procédé ou de service susceptibles de transformer profondément leur travail à leur bénéfice et à celui des usagers. Dans certains cas, ils sont même en mesure de les réaliser eux-mêmes en exploitant la pléthore de services offerts par les plateformes, en matière de communication, de coopération, de gestion documentaire, de promotion, de gestion de base de données en mode SAAS (Software As A Service) et même de paiement.

Pour imaginer ces solutions, ils ne sont pas seuls. Ils peuvent s'appuyer sur des communautés externes de passionnées, qui sont soit dans une logique d'usage de nouveaux produits commerciaux (Dropbox, Wathsapp, Google Drive, réseaux sociaux, etc...), soit de produits Open Source, soit d'invention de nouvelles pratiques professionnelles partagées au sein de communautés de pratique internes à l'organisation. Cette dimension transgressive de certains usages du numérique (cf. infra) n'est pas sans susciter des conflits avec les directions des systèmes d'information (DSI). Dans certaines organisations, il existe un directeur du digital (Chief Digital Officer ou Directeur de la Stratégie Digitale) distincte de la DSI qui est en charge d'accompagner la transition numérique avec des moyens propres. Dans d'autres cas, c'est à l'intérieur de la DSI que des équipes sont dédiées à la transition, équipes qui fonctionnent sur un mode plus agile que les autres projets.

\section{5-L'innovation collaborative et le paradoxe de l'innovation par « transgression régulée »}

Ce caractère en partie déviant de l'innovation avait déjà été mis en évidence dans les travaux sur l'innovation ordinaire de N. Alter (2010). Dans le secteur public, l'innovation fait d'abord référence à la conception et au design de nouveaux services au bénéfice des usagers. Une recherche récente a montré l'importance de ces démarches qui touchent de près ou de loin quasiment toutes les entreprises publiques et les administrations (Weller, Pallez 2017). Mais elle concerne également la mise en place de nouveaux processus interne impactant directement le travail. 
L'innovation collaborative se déploie dans ce contexte d'accélération de la transformation numérique qui a lui-même fortement contribué à rendre possible la mondialisation sans précédent des échanges. Elle rend également possible la "dématérialisation» et la " désintermédiation », en faisant progressivement basculer les organisations dans un régime d'innovation intensive qui les transforme en "métabolismes génératifs, producteurs de création collective, de nouvelles formes d'entreprises et de collectifs solidaires » (Hatchuel et al., 2017).

Pour faire face à ces changements, de nombreuses organisations mettent en place de nouveaux dispositifs qui étaient jusqu'alors plutôt le propre des milieux militants investis dans des tiers lieux, makers spaces, living-labs, pour ne prendre que quelques exemples (Berrebi-Hoffmann, Bureau, Lallement 2018). Ces nouveaux dispositifs correspondent à la mise en place de démarches «d'innovation collaborative » que nous n'assimilons pas à l'innovation ouverte. L'innovation collaborative, que l'on peut aussi appeler design collaboratif, correspond selon nous à des activités collectives de conception basées sur des principes de coopération et de participation stimulant la créativité, la résolution de problème et la prise en compte des partie prenantes.

Dans l'innovation collaborative, la coopération s'inscrit généralement dans le régime de la coopération spontanée (Zacklad, 2015) correspondant à des groupes de travail libres en mesure d'attendre des objectifs très variés sur des périodes généralement assez courtes. L'animation de ces groupes relève des principes et des méthodes du « design de relation » (Zacklad, 2017) qui doivent garantir un déroulement de l'activité satisfaisant aux plans émotionnel et cognitif. Comme nous l'avons évoqué plus haut, ces démarches empruntent elles-mêmes, selon les contextes, aux méthodes agiles, au design-thinking, ou aux applications de la théorie C\&K, pour ne prendre que quelques exemples.

La participation concerne la composition des groupes, l'identification des membres légitimes, de leurs droits et devoirs. Elle vise à rassembler un nombre suffisant de parties prenantes pour éclairer la conception en garantissant la pertinence des contributions et l'engagement dans la durée. Il est souvent nécessaire d'associer à la fois des membres détenteurs de compétences spécialisées pour stimuler les propositions, des représentants des bénéficiaires pour évaluer leur pertinence, mais aussi, à un titre ou à un autre, des décideurs, les "sponsors ", en mesure de garantir au projet des ressources suffisantes.

Ces caractéristiques permettent de différencier l'innovation collaborative de l'innovation ouverte. La première implique une définition attentive des participants, une réflexion sur la dynamique des échanges et elle peut être interne aux organisations autant qu'externe. Par contraste, l'innovation ouverte est essentiellement tournée vers les apports externes et elle peut aussi s'appuyer sur des «foules anonymes » via des plateformes en mode crowdsourcing.

Cette nouvelle configuration correspond au passage du stade de l'innovation ordinaire décrite par Alter (2010), dans laquelle les organisations restent caractérisées par la recherche d'ordre et de régularité et qui tolère la déviance inventive parce qu'elle pourra ensuite la re-normaliser, au stade de l'innovation par la «transgression régulée » (Zacklad à paraître). A ce stade, pour faire face aux exigences de renouvellement rapide de leurs offres et de leurs procédés, les organisations encouragent la mise en place de nouveaux dispositifs qui plongent les équipes dans des contextes permettant de faciliter la création, les échanges transversaux et les nouvelles manières travailler en groupe et de manager les équipes. Elles sont conscientes de la nécessité de « casser les codes » et sont donc sont prêtes à donner des espaces de liberté même si cela 
implique de faire émerger des propositions et des rencontres qui étaient jusqu'alors proscrites. Tout en étant garant de l'ordre, le haut management incite lui-même à une forme de désordre, non sans placer parfois les collaborateurs dans un système de « double contrainte » complexe.

La généralisation des dispositifs d'innovation collaborative est caractéristique de cette époque. Ces dispositifs peuvent prendre différentes formes : déroulement de sessions de co-design pour faciliter l'innovation, investissement d'espaces de type tiers lieux permettant la rencontre avec d'autres communautés, développement de laboratoires d'innovation internes permettant la mise en place, dans des espaces permanents ou éphémères, de démarche de codesign (Foliot et al., 2019).

Un des enjeux principaux de l'innovation collaborative, même s'il n'est généralement pas complètement explicité, est de former les équipes au travail «conception », conception de produits, de services, d'organisation, d'applications numériques, etc. et ce à tous les niveaux des organisations. Cette démarche est difficile car, hors des formations initiales aux métiers créatifs d'architecte, de designer, d'ingénieur-maker, de spécialiste du numérique, de rédacteurcommunicant, de chercheur, etc., les travailleurs ne sont pas formés à ces formes de raisonnement même si la culture du projet s'est largement répandue.

Les labs d'innovation internes, comme ceux de Pôle Emploi décrits dans cet ouvrage (voir le chapitre de S. Rocq et L. Remaud) incarnent de la manière la plus forte les changements culturels liés à l'innovation collaborative dans le contexte de la « transgression régulée ». Mais leurs succès dans la longue durée ne sont jamais garantis dans les grandes organisations car les phases de conception n'ont qu'un temps, elles s'arrêtent au moment où le plan, la maquette ou le prototype sont au point. En effet, de nombreuses organisations sont également des univers de "mise à l'échelle" des solutions générées dans les Labs, dans le service comme dans l'industrie.

\section{Conclusion}

La transition numérique et l'innovation collaborative sont intimement liées, l'innovation collaborative étant la plupart du temps une condition nécessaire à la réussite des actions de transformation numérique comme on le voit dans l'exemple de la gendarmerie qui à l'instar de nombreuses autres administrations s'appuie sur un lab d'innovation pour développer la tablette Néo et sa bibliothèque d'applications (voir le chapitre de $\mathrm{M}$. Fumery). Le magazine «acteurspublics» ( ${ }^{\circ} 138$, Mars-Avril 2019) a ainsi recensé près de 60 laboratoires d'innovation publique dans les ministères, les services déconcentrés, les collectivités territoriales, la santé et les opérateurs ou entités indépendantes, labs qui dans leur grande majorité contribuent à la conception d'applications numériques au bénéfice des usagers et des agents.

Si ces démarches correspondent aussi parfois à des slogans et des invocations qui sont utilisés de manière abusive, elles correspondent selon nous aux prémices d'une mutation importante de la manière dont l'environnement de travail et la nature des services rendus au public tendent à être redéfinis avec la participation des agents à tous les niveaux des organisations.

\section{Bibliographie}

Alter N., L’innovation ordinaire (3e éd). Paris, PUF, 2010. 
Berrebi-Hoffmann, I., Bureau, M.-C., \& Lallement, M. Makers: enquête sur les laboratoires du changement social. Paris, France : Éditions du Seuil, 2018.

Bobillier-Chaumon M.-E., Du rôle des TIC dans la transformation digitale de l'activité et de la santé au travail. Revue des conditions de travail, (6), 16-24, 2017. Repéré à https://www.anact.fr/durole-des-tic-dans-la-transformation-digitale-de-lactivite-et-de-la-sante-au-travail

Falzon P., "Pour une ergonomie constructive», in Ergonomie constructive, Paris, Presses Universitaires de France, p. 1-16, 2013.

Foliot, C., Serikoff, G., \& Zacklad, M. (2019). Le lab des labs. https://hal.archives-ouvertes.fr/hal02437318

Hatchuel A., Masson P. L., Weil, B., « De la gestion des connaissances aux organisations orientées conception», Revue internationale des sciences sociales, (171), 29-42, 2002. https://doi.org/10.3917/riss.171.0029

Hatchuel A., Masson P. L., Weil B., « Comprendre et soutenir l'innovation contemporaine : théorie de la conception et métabolisme des nouveaux collectifs », La lettre de l'InSHS, 2017.

Vygotski L. S., Pensée et langage. Paris, Ed. sociales, 1992.

Weller J.-M., Pallez F., « Les formes d'innovation publique par le design : un essai de cartographie », Sciences du Design, $n^{\circ}$ 5(1), 32-51, 2017. Repéré à https://www.cairn.info/revue-sciences-dudesign-2017-1-page-32.htm

Zacklad M., " Une théorisation communicationnelle et documentaire des TIC », in C. Brossaud, B. Reber (Éds), Socio-informatique et démocratie cognitive, Paris, Hermès science publicationsLavoisier, p. 20-35, 2007. Repéré à https://archivesic.ccsd.cnrs.fr/sic 00180186

Zacklad M., "Vers une informatique au service de l'homme », Personnel, (527), 63-65, 2012. Repéré

àhttps://www.academia.edu/31069639/Vers_une informatique_au_service_de lhomme

Zacklad M., «La transition numérique : impact sur le travail et son management ", communication présentée à l'Atelier-débat du club Innovation \& Management, Paris, 2017. Repéré à https://www.slideshare.net/ManuelZacklad/transition-numerique-139521379

Zacklad $\mathrm{M}$, «Les laboratoires d'innovation internes et la transgression régulée », ATIC, (2), à paraître. 